\title{
Association Between Hospital Volume, Therapy Types, and Overall Survival in Stage III and IV Cutaneous Malignant Melanoma
}

Sri Harsha Tella, MD,*; Anuhya Kommalapati, MBBS ${ }^{a, b, *} ;$ Apar Kishor Ganti, MD,d; and Alissa S. Marr, MD

\section{ABSTRACT}

Background: The advent of targeted therapies and immunomodulatory agents has revolutionized the management of advanced cutaneous malignant melanoma (MMel) by prolonging overall survival. This study evaluated the therapeutic and survival disparities among patients with advanced MMel based on hospital volume using the National Cancer Database (NCDB). Methods: A retrospective analysis using regression models and Kaplan-Meier estimates was performed from the data obtained from the NCDB on patients with MMel diagnosed in 2004 through 2015. Results: A total of 40,676 patients with MMel were treated at 1,260 facilities. Multivariable analysis showed that facility volume was an independent predictor of overall survival $(P<.0001)$. Compared with patients treated at high-volume facilities (tertile 3 [T3]), those with stage III disease $(n=27,528)$ treated at intermediate- and low-volume facilities (T2 and $\mathrm{T} 1$, respectively) had a significantly higher risk of death (T2 hazard ratio $[\mathrm{HR}], 1.15 ; 95 \% \mathrm{Cl}, 1.09-1.20 ; \mathrm{T} 1 \mathrm{HR}, 1.23 ; 95 \% \mathrm{Cl}$, 1.17-1.29). Compared with patients treated at $\mathrm{T} 3$ facilities, those with stage IV disease $(n=13,148)$ treated at lower-tertile facilities had a significantly higher risk of death $(\mathrm{T} 2 \mathrm{HR}, 1.16$; $95 \% \mathrm{Cl}, 1.10-1.21$; T1 HR, 1.29; 95\% Cl, 1.23-1.36). Further, patients with stage IV disease treated at T3 facilities (vs T1 facilities) were more likely to receive chemotherapy (38\% vs $28 \%$ ) and immunotherapy $(23 \%$ vs 10\%) $(P<.0001)$. Conclusions: Patients with advanced-stage MMel treated at high-volume facilities had significantly improved survival and were more likely to receive chemotherapy and immunotherapy. J Natl Compr Canc Netw 2019;17(11):1334-1342 doi: $10.6004 /$ jnccn.2019.7320

aDepartment of Internal Medicine, University of South Carolina School of Medicine, Columbia, South Carolina; ${ }^{b}$ Department of Oncology, H. Lee Moffitt Cancer Center, Tampa, Florida; and 'Department of Hematology and Oncology, University of Nebraska Medical Center, and 'VA Nebraska Western lowa Health Care System, Omaha, Nebraska.

*These authors contributed equally and are co-first authors.

\section{Background}

Cutaneous malignant melanoma (MMel) constitutes approximately $1 \%$ of all skin cancers, with an incidence of approximately 22.3 cases $/ 100,000$ personyears in the United States. ${ }^{1}$ The mortality rate is 2.7 deaths/100,000 persons, with most deaths occurring in patients with advanced $\mathrm{MMel}$ (ie, regional and/or distant metastases). ${ }^{1}$ Management of advanced MMel (stages III and IV) often involves the coordinated use of a multimodality approach and effective handling of toxicities. In the past few years, the advent of targeted therapies such as tyrosine kinase inhibitors and immunomodulatory agents has revolutionized the management of advanced MMel by prolonging overall survival (OS). ${ }^{2}$ However, these newer modalities are also known to be associated with unique toxicities. Management of these toxicities requires an experienced comprehensive care team, which may play a significant role in not only improving quality of life but also prolonging survival in patients with MMel. Given the heterogeneity of stage III and IV presentations and the involvement of multimodality therapies, these patients may benefit from management in facilities with comprehensive care teams with greater clinical expertise. Previous studies have described how greater hospital volume and provider experience are associated with improved survival in solid organ malignancies. ${ }^{3}$ This study analyzed the association between facility volume, treatment modalities offered, and different risk-adjusted outcomes in patients with advanced (stage III and IV) MMel.

\section{Methods}

\section{Data Source}

Data from 2004 through 2015 were obtained from the National Cancer Database (NCDB). Because both facility and patient data are deidentified in the NCDB, the University of South Carolina Institutional Review Board exempted this study from review.

\section{Study Population}

A total of 523,492 cutaneous MMel cases diagnosed in 2004-2015 were identified in the NCDB. Because the 
NCDB provides data based on the facility where the malignancy was initially diagnosed and first-line treatment was offered, patients diagnosed at one facility and treated at another facility were excluded $(n=3,788)$. Patients with multiple malignancies $(\mathrm{n}=101,746)$ and those with stage I or II disease $(n=239$ and 500 , respectively) were excluded. Cases diagnosed in 2015 $(n=5,246)$ were also excluded from OS analysis to allow at least a 1-year follow-up. To minimize missing data, reference date cutoff of 1 was used so that the facilities were accountable for the completeness of the data. The case selection process is detailed in Figure 1.

Facility Volume, Therapeutics, and Survival Analysis Patients in the study cohort were divided into tertiles based on average annual patient volume treated at a facility (low-volume facility, lower-third tertile [T1]; intermediate-volume facility, middle-third tertile [T2]; high-volume facility, higher-third tertile [T3]). The primary outcome of interest was the time interval between stage III and IV cutaneous MMel diagnosis and death. Patients who were alive at last follow-up were censored. NCDB-specific definitions of various treatment modalities (chemotherapy, immunotherapy, and/or surgery) can be obtained from the NCDB participant user file data dictionary (http://ncdbpuf.facs.org/v-terms-dd/86).

\section{Covariables Included}

The primary predictor of interest in the analysis was facility volume. Patients in the tertiles were described based on demographics (age, sex, race), insurance type (no insurance, private, Medicare, Medicaid, other/ unknown), Charlson-Deyo comorbidity index score, lactate dehydrogenase (LDH) level (in stage IV disease only), household income, education status, geographic region, histology, disease site, and type of therapy received (surgery, chemotherapy, immunotherapy). These covariables were included in the multivariable analysis.

\section{Statistical Analysis}

SPSS Statistics, version 24 (IBM Corp) accounted for the clustering of patients within facilities (based on volume: T1, T2, and T3 facilities), and a multivariable Cox proportional hazards regression model was used to determine the volume-outcome relationship after adjusting for the covariables. Nonnormally distributed continuous variables were expressed as median and range. One-way analysis of variance was used to compare continuous variables, and the chi-square test was used to compare categorical variables. OS was estimated using the Kaplan-Meier method and log-rank tests. A $P$ value of $<.05$ was considered statistically significant.

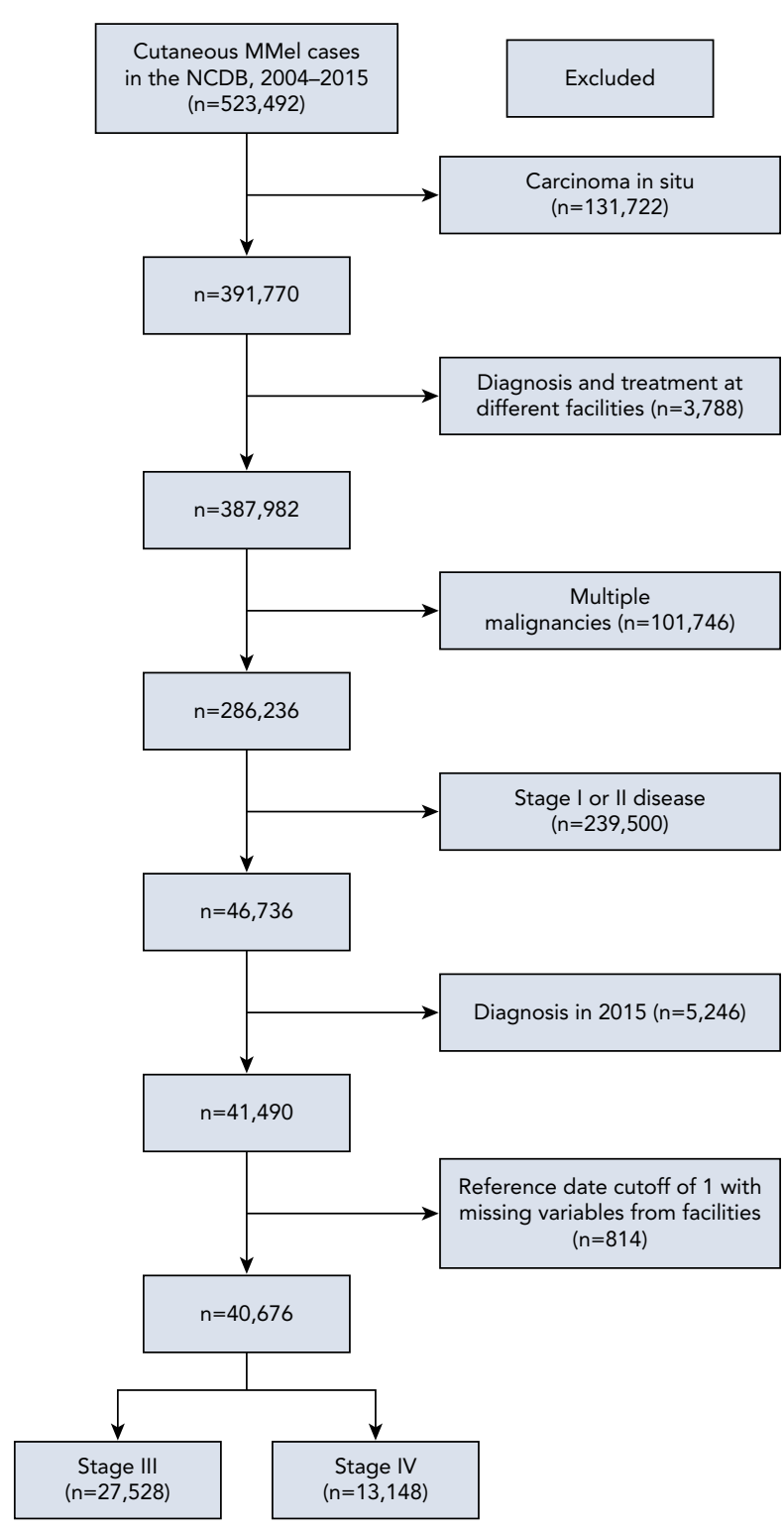

Figure 1. CONSORT diagram for case selection.

Abbreviations: MMel, malignant melanoma; NCDB, National Cancer Database.

\section{Results}

Baseline Characteristics and Distribution by Facility Volume

\section{Stage III Disease}

A total of 27,528 patients with stage III cutaneous MMel treated at 1,260 facilities met the inclusion criteria. Median age at diagnosis was 57 years (range, age $18-90$ years), and $62 \%$ were male. Median annual facility volume was 9 patients/year (range, $1-120$ patients/ year). Facilities were classified by tertile (mean patients with stage III cutaneous MMel treated per year): $\mathrm{Tl}$ facilities, $\leq 5$ cases/year; T2 facilities, 6 to 18 cases/year; 
and T3 facilities, $\geq 19$ cases/year. T1, T2, and T3 facilities accounted for $32 \%(n=8,848), 35 \%(n=9,560)$, and $33 \%(n=9,120)$ of the cases, respectively. Significant differences in patient demographics and tumor characteristics were noted with respect to hospital volume (Table 1). Compared with patients treated at $\mathrm{T} 1$ facilities, those treated at T3 facilities tended to have head and neck disease (18\% vs $13 \%)$, had higher median household income ( $37 \%$ vs $33 \%$ ), were more highly educated ( $29 \%$ vs $25 \%$ ), and had private health insurance $(59 \%$ vs $51 \%) \quad(P<.0001$ for all comparisons).

\section{Stage IV Disease}

A total of 13,148 patients with stage IV cutaneous MMel treated at 1,247 facilities met the inclusion criteria. Median age at diagnosis was 62 years (range, age $18-90$ years), and $67 \%$ were male. Median annual facility volume was 5 patients/year (range, 1-120 patients/year). The facilities were classified by tertile (mean patients with stage IV cutaneous MMel treated/ year): T1 facilities, $\leq 3$ cases/year; T2 facilities, 4-9 cases/year; and T3 facilities, $\geq 10$ cases/year, respectively. T1, T2, and T3 facilities accounted for $32 \%$ $(n=4,205), 35 \%(n=4,606)$, and $33 \%(n=4,337)$ of the cases, respectively. Significant differences in patient demographics and tumor characteristics were noted with respect to hospital volume (Table 2). Compared with patients treated at $\mathrm{T} 1$ facilities, those treated at T3 facilities tended to have head and neck disease ( $10 \%$ vs $8 \%$ ), had higher median household income ( $36 \%$ vs $33 \%$ ), were more highly educated ( $28 \%$ vs $25 \%$ ), and had private health insurance ( $48 \%$ vs $36 \%$ ) $(P<.0001)$.

\section{Treatment Analysis Based on Facility Volume}

\section{Stage III Disease}

No clinically meaningful differences in therapy patterns were noted among the T1, T2, and T3 facilities. Surgery ( $91 \%$ vs $93 \%)$, chemotherapy ( $10 \%$ vs $7 \%$ ), and immunotherapy (26\% vs $27 \%$ ) patterns were similar between the T1 and T3 facilities, respectively. Similar therapeutic patterns were also noted in T2 facilities (Table 1).

\section{Stage IV Disease}

Significant differences were identified in the management of stage IV cutaneous MMel by facility volume. Patients at T3 facilities were more likely to receive systemic treatment $(69 \%, 74 \%$, and $83 \%$ at $\mathrm{T} 1, \mathrm{~T} 2$, and T3 facilities, respectively; $P<.0001$ ). Compared with patients treated at $\mathrm{T} 1$ facilities, those treated at $\mathrm{T} 3 \mathrm{fa}-$ cilities were more likely to receive chemotherapy $(38 \%$ vs $28 \%$ ) and immunotherapy ( $23 \%$ vs $10 \%)(P<.0001)$.
Surgical therapy patterns were similar among facilities ( $29 \%$ for $\mathrm{T} 1$ and $\mathrm{T} 2$ vs $31 \%$ for T3) (Table 2 ).

\section{Survival Analysis}

\section{Stage III Disease}

The unadjusted median OS by facility volume was 77 months for T1 facilities, 92 months for T2 facilities, and 136 months for T3 facilities $(P<.001)$; patients treated at T3 facilities appeared to live approximately 5 years longer than those treated at T1 facilities. Furthermore, patients treated at T3 facilities had significantly better 1-, 3-, and 5-year OS compared with those treated at T1 facilities $(P<.001)$ (Table 3, Figure $2 \mathrm{~A})$. Cox multivariable analysis showed that female sex, white race, private insurance, higher household income, lower Charlson-Deyo comorbidity index score, primary disease site of limbs, higher hospital volume, and receipt of surgery and/or immunotherapy were associated with better survival (Table 4). Interestingly, receipt of chemotherapy was inversely associated with OS (hazard ratio $[\mathrm{HR}], 1.41 ; 95 \% \mathrm{CI}, 1.32-1.50 ; P<.001$ ), whereas immunotherapy was not associated with OS benefit. After adjusting for all covariables, higher facility volume ( $\geq 19$ cases/year) was an independent predictor of better OS. Compared with patients treated at T3 facilities, those treated at $\mathrm{T} 1$ facilities had a significantly higher risk of death (T2: HR, 1.15; 95\% CI, 1.09-1.20; T1: HR, 1.23; 95\% CI, 1.17-1.29).

\section{Stage IV Disease}

The unadjusted median OS by facility volume was 6 months for T1 facilities, 8 months for T2 facilities, and 11 months for T3 facilities $(P<.001)$. Patients treated at T3 facilities had a significantly better 1-, 3-, and 5-year OS compared with those treated at $\mathrm{T} 1$ facilities $(P<.001)$ (Table 3, Figure 2B). Cox multivariable analysis showed that female sex, white race, private insurance, higher household income, lower Charlson-Deyo comorbidity index score, lower LDH level, primary disease site of limbs, higher facility volume, and receipt of surgery and/or immunotherapy were associated with better survival (Table 4). After adjusting for all covariables, facility volume was independently associated with all-cause mortality. Compared with patients treated at T3 facilities, those treated at T2 and T1 facilities had a significantly higher risk of death (T2: HR, 1.16; 95\% CI, 1.10-1.21; T1: HR, 1.29; 95\% CI, 1.23-1.36). Receipt of surgery and/or immunotherapy was independently associated with prolonged OS $(P<.001)$. Because the FDA approved immune and targeted therapeutic agents such as ipilimumab and vemurafenib in 2011, we analyzed the median OS difference before (2004-2010) and after 2011 (2012-2015) in T3 
Table 1. Characteristics of Patients With Stage III Cutaneous Malignant Melanoma

\begin{tabular}{|c|c|c|c|c|c|}
\hline & & & tile/Hospital Volume (cas & & \\
\hline & Overall & T1/Low (1-5) & T2/Intermediate (6-18) & T3/High ( $\geq 19)$ & P Value \\
\hline Total & 27,528 & $8,848(32 \%)$ & $9,560(35 \%)$ & $9,120(33 \%)$ & \\
\hline Median age (range), y & $57(18-90)$ & $58(18-90)$ & $57(18-90)$ & $56(18-90)$ & $<.001$ \\
\hline Male sex, \% & 62 & 63 & 61 & 61 & .04 \\
\hline Race, \% & & & & & .07 \\
\hline Black & 1 & 1 & 1 & 1 & \\
\hline Other & 2 & 2 & 2 & 2 & \\
\hline Insurance status, \% & & & & & $<.0001$ \\
\hline No insurance & 5 & 6 & 5 & 4 & \\
\hline Private & 55 & 51 & 56 & 59 & \\
\hline Median household income, \% & & & & & $<.0001$ \\
\hline$<\$ 38,000 / y$ & 14 & 14 & 14 & 14 & \\
\hline$\$ 38,000-\$ 47,999 / y$ & 23 & 24 & 22 & 23 & \\
\hline$\$ 48,000-\$ 62,999 / y$ & 27 & 29 & 27 & 26 & \\
\hline$\geq \$ 63,000 / y$ & 36 & 33 & 37 & 37 & \\
\hline Education status, ${ }^{\text {, }} \%$ & & & & & $<.0001$ \\
\hline$\geq 21.0$ & 13 & 14 & 13 & 12 & \\
\hline $13.0-20.9$ & 24 & 25 & 25 & 23 & \\
\hline $7.0-12.9$ & 35 & 36 & 33 & 35 & \\
\hline$\leq 7.0$ & 28 & 25 & 29 & 29 & \\
\hline Geographic region, \% & & & & & $<.0001$ \\
\hline $2-3$ & 3 & 3 & 3 & 2 & \\
\hline Histology, \% & & & & & $<.0001$ \\
\hline Nodular & 22 & 23 & 21 & 22 & \\
\hline Superficial spreading & 21 & 17 & 19 & 29 & \\
\hline Acral lentiginous & 3 & 2 & 2 & 4 & \\
\hline Other & 54 & 58 & 58 & 45 & \\
\hline Disease site, \% & & & & & $<.0001$ \\
\hline Head and neck & 16 & 13 & 17 & 18 & \\
\hline Trunk & 34 & 34 & 34 & 34 & \\
\hline Limbs & 42 & 43 & 43 & 41 & \\
\hline Other sites & 8 & 10 & 6 & 7 & \\
\hline Therapy received, \% & & & & & $<.0001$ \\
\hline None & 3 & 4 & 2 & 3 & \\
\hline Surgery & 93 & 91 & 94 & 93 & \\
\hline Chemotherapy & 8 & 10 & 8 & 7 & \\
\hline Immunotherapy & 27 & 26 & 27 & 27 & \\
\hline
\end{tabular}

a $P$ value signifies any statistical differences among the low-, intermediate-, and high-volume facilities in baseline characteristics.

bPercentage of patients who did not graduate high school. 
Table 2. Characteristics of Patients With Stage IV Cutaneous Malignant Melanoma

\begin{tabular}{|c|c|c|c|c|c|}
\hline & & & ile/Hospital Volume (cas & & \\
\hline & Overall & T1/Low (1-3) & T2/Intermediate (4-9) & T3/High ( $\geq 10)$ & $P$ Value $^{\mathrm{a}}$ \\
\hline Total & 13,148 & $4,205(32 \%)$ & $4,606(35 \%)$ & 4,337 (33\%) & \\
\hline Number of facilities & 1,247 & 830 & 320 & 97 & \\
\hline Median age (range), y & $62(18-90)$ & $64(18-90)$ & $63(18-90)$ & $60(18-90)$ & $<.0001$ \\
\hline Male sex, \% & 67 & 66 & 68 & 68 & .04 \\
\hline Race, \% & & & & & .004 \\
\hline White & 96 & 96 & 96 & 95 & \\
\hline Black & 2 & 2 & 2 & 2 & \\
\hline Other & 2 & 2 & 2 & 3 & \\
\hline Insurance status, \% & & & & & $<.0001$ \\
\hline No insurance & 7 & 8 & 7 & 5 & \\
\hline Private & 41 & 36 & 39 & 48 & \\
\hline Medicaid & 9 & 8 & 9 & 9 & \\
\hline Medicare & 41 & 45 & 42 & 34 & \\
\hline Other & 2 & 3 & 3 & 4 & \\
\hline Median household income, \% & & & & & $<.0001$ \\
\hline$<\$ 38,000 / y$ & 15 & 15 & 16 & 13 & \\
\hline$\$ 38,000-\$ 47,999 / \mathrm{y}$ & 24 & 25 & 25 & 23 & \\
\hline$\$ 48,000-\$ 62,999 / y$ & 28 & 27 & 28 & 28 & \\
\hline$\geq \$ 63,000 / y$ & 33 & 33 & 31 & 36 & \\
\hline Education status, ${ }^{b} \%$ & & & & & $<.001$ \\
\hline$\geq 21.0$ & 15 & 16 & 17 & 13 & \\
\hline $13.0-20.9$ & 26 & 25 & 26 & 25 & \\
\hline $7.0-12.9$ & 33 & 34 & 31 & 34 & \\
\hline$\leq 7.0$ & 26 & 25 & 26 & 28 & \\
\hline Geographic region, \% & & & & & .39 \\
\hline Metropolitan & 79 & 78 & 80 & 79 & \\
\hline Urban & 15 & 15 & 15 & 14 & \\
\hline Rural & 2 & 2 & 2 & 2 & \\
\hline Unknown & 4 & 5 & 3 & 5 & \\
\hline Charlson-Deyo comorbidity index score, \% & & & & & $<.0001$ \\
\hline $0-1$ & 93 & 92 & 93 & 95 & \\
\hline $2-3$ & 7 & 8 & 7 & 5 & \\
\hline Histology, \% & & & & & .001 \\
\hline Nodular & 8.4 & 7.3 & 8.7 & 9.3 & \\
\hline Superficial spreading & 3 & 2 & 3 & 3 & \\
\hline Acral lentiginous & 0.6 & 0.7 & 0.3 & 0.7 & \\
\hline Other & 88 & 90 & 89 & 87 & \\
\hline Site, $\%$ & & & & & .001 \\
\hline Head and neck & 9 & 8 & 9 & 10 & \\
\hline Trunk & 14 & 14 & 13 & 14 & \\
\hline Limbs & 14 & 14 & 13 & 14 & \\
\hline Other sites & 63 & 63 & 65 & 62 & \\
\hline Therapy received & & & & & $<.0001$ \\
\hline None & 25 & 31 & 26 & 17 & \\
\hline Surgery & 30 & 29 & 29 & 31 & \\
\hline Chemotherapy & 32 & 28 & 31 & 38 & \\
\hline Immunotherapy & 15 & 10 & 12 & 23 & \\
\hline
\end{tabular}

a $P$ value signifies any statistical differences among the low-, intermediate-, and high-volume facilities in baseline characteristics.

bPercentage of patients who did not graduate high school. 


\begin{tabular}{|lcccc|}
\hline \multirow{5}{*}{$\begin{array}{c}\text { Table 3. Survival of Patients With Stage III and IV } \\
\text { Cutaneous }\end{array}$} \\
Facility & $\begin{array}{c}\text { Median Survival } \\
\text { (mo) }\end{array}$ & \multicolumn{4}{c|}{ Overall Survival (\%) } \\
\cline { 3 - 6 } & $\mathbf{1 - y}$ & $\mathbf{3 - y}$ & $\mathbf{5 - y}$ \\
\hline Stage III disease & 77 & 89 & 66 & 54 \\
\hline T1 & 92 & 92 & 69 & 58 \\
\hline T2 & 136 & 93 & 74 & 63 \\
\hline T3 & & & & \\
\hline Stage IV disease & 6 & 34 & 17 & 13 \\
\hline T1 & 8 & 39 & 20 & 15 \\
\hline T2 & 11 & 47 & 24 & 19 \\
\hline T3 & & & & \\
\hline
\end{tabular}

Abbreviations: T1, low-volume; T2, intermediate-volume; T3, high-volume.

facilities and found that the median OS for patients with stage IV disease managed at T3 facilities after 2011 was significantly better than for those treated before 2011 (9.8 vs 13 months; $P<.001$ ).

\section{Discussion}

In the past decade, several studies have evaluated the relationship between facility volume and survival outcomes in the field of surgical oncology. ${ }^{4}$ Given the advent of newer therapies and the increased understanding of tumor biology and molecular markers, medical management of melanoma has become more complex, often necessitating a comprehensive care team involving multiple subspecialties. With this increased complexity of care, patients may be referred more frequently to specialized high-volume (T3) facilities that can coordinate management among different subspecialties. Recent studies on the volume-outcome relationship in the medical management of solid organ

A

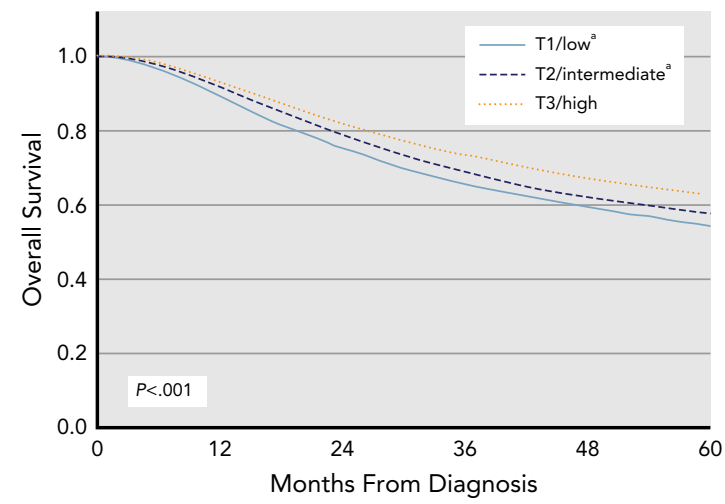

malignancies have shown promising results., ${ }^{5,6}$ On the contrary, studies involving some advanced-stage malignancies, such as hepatocellular carcinoma, have shown no clinically meaningful survival benefit associated with treatment at high-volume facilities. ${ }^{7}$ In this retrospective analysis, using a national cancer dataset, we showed that patients with advanced (stages III and IV) cutaneous MMel treated at T3 facilities had lower mortality than those treated at $\mathrm{T} 1$ facilities, after adjusting for patient demographics, functional status, tumor characteristics, and therapy received. The difference was much more pronounced in stage III disease, with an almost 5 -year median OS difference between T1 and T3 facilities.

Significant socioeconomic disparities existed in the baseline characteristics of the patients managed at the range of facilities in our cohort. Patients with private health insurance, greater education, and higher median household income were more likely to be treated at T3 facilities. Low household income, lack of health insurance, and less education have all been found to be predictors for suboptimal cancer care. This association between socioeconomic status and cancer outcomes is well established in a few population-based studies. ${ }^{8-10}$ However, we demonstrated the OS benefit of receiving treatment at T3 facilities even after adjusting for these socioeconomic factors. Moreover, previous reports have shown that patients with a higher socioeconomic status did not have significantly improved survival rates for poorprognosis cancers. ${ }^{11}$

Our findings showed a significant difference in the rates of administration of chemotherapy ( $38 \%$ vs $28 \%$ ) and immunotherapy ( $23 \%$ vs $10 \%$ ) in stage IV disease between T3 and T1 facilities, respectively. Indeed, this study showed that chemotherapy did not confer an
B

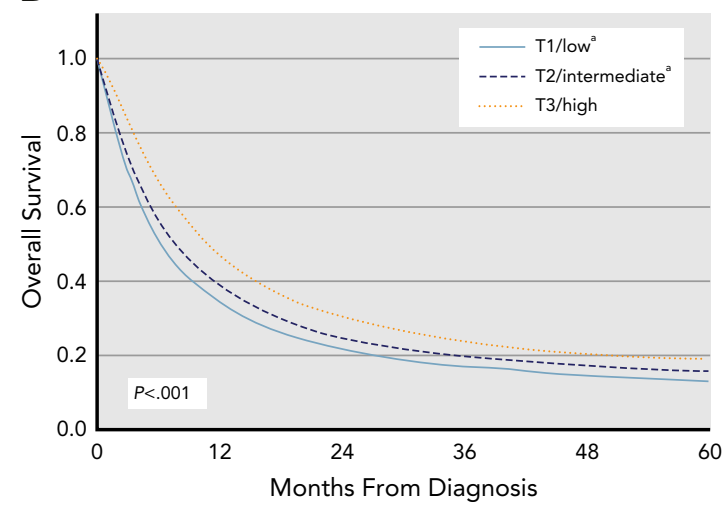

Figure 2. Comparison of unadjusted overall survival curves of (A) stage III and (B) stage IV cutaneous malignant melanoma based on annual facility volumes.

a $P$ value vs that of a high-volume facility $(P<.001)$. 
Table 4. Cox Multivariable Analysis to Predict Overall Mortality

\begin{tabular}{|c|c|c|c|c|}
\hline \multirow[b]{2}{*}{ Parameter } & \multicolumn{2}{|c|}{ Stage III } & \multicolumn{2}{|c|}{ Stage IV } \\
\hline & HR $(95 \% \mathrm{Cl})$ & $P$ Value & HR (95\% Cl) & $P$ Value \\
\hline Age & $1.027(1.026-1.029)$ & $<.001$ & $1.008(1.006-1.010)$ & $<.01$ \\
\hline \multicolumn{5}{|l|}{ Sex (female, ref) } \\
\hline Male & $1.28(1.23-1.33)$ & $<.001$ & $1.11(1.06-1.16)$ & $<.01$ \\
\hline \multicolumn{5}{|l|}{ Race (white, ref) } \\
\hline Black & $1.31(1.12-1.53)$ & $<.001$ & $1.14(0.99-1.32)$ & .06 \\
\hline \multicolumn{5}{|c|}{ Insurance status (private, ref) } \\
\hline Uninsured & $1.48(1.36-1.62)$ & $<.001$ & $1.44(1.32-1.56)$ & $<.001$ \\
\hline Medicaid & $1.84(1.69-2.01)$ & & $1.46(1.34-1.57)$ & \\
\hline Medicare & $1.24(1.17-1.31)$ & & $1.16(1.10-1.22)$ & \\
\hline \multicolumn{5}{|c|}{ Household income $(\geq \$ 63,000 / y$, ref) } \\
\hline$<\$ 38,000 / y$ & $1.26(1.17-1.36)$ & $<.001$ & $1.09(1.02-1.16)$ & $<.001$ \\
\hline$\$ 38,000-\$ 47,999 / y$ & $1.11(1.04-1.18)$ & & $1.09(1.04-1.15)$ & \\
\hline$\$ 48,000-\$ 62,999 / y$ & $1.08(1.02-1.14)$ & & $1.13(1.08-1.19)$ & \\
\hline \multicolumn{5}{|c|}{ Charlson-Deyo comorbidity index score $(0-1$, ref) } \\
\hline $2-3$ & $1.57(1.43-1.74)$ & $<.001$ & $1.57(1.46-1.69)$ & $<.001$ \\
\hline \multicolumn{5}{|c|}{ Primary disease site (limbs, ref) } \\
\hline Head and neck & $1.30(1.23-1.37)$ & $<.001$ & $0.92(0.84-1.003)$ & .06 \\
\hline Trunk & $1.19(1.14-1.25)$ & & $1.24(1.15-1.34)$ & $<.001$ \\
\hline \multicolumn{5}{|l|}{ LDH level (normal, ref) } \\
\hline $1.5 \times$ ULN & $0.99(0.89-1.11)$ & .441 & $1.14(1.03-1.26)$ & $<.001$ \\
\hline $1.6-10 \times$ ULN & $1.09(0.88-1.36)$ & & $1.71(1.53-1.92)$ & \\
\hline$>10 \times$ ULN & $1.21(0.83-1.76)$ & & $1.98(1.68-2.34)$ & \\
\hline \multicolumn{5}{|c|}{ Hospital volume (high [T3], ref) } \\
\hline Low (T1) & $1.23(1.17-1.29)$ & $<.001$ & $1.29(1.23-1.36)$ & $<.001$ \\
\hline Intermediate (T2) & $1.15(1.09-1.20)$ & & $1.16(1.10-1.21)$ & \\
\hline \multicolumn{5}{|c|}{ Therapy received (none, ref) } \\
\hline Surgery & $0.77(0.68-0.88)$ & $<.001$ & $0.59(0.58-0.63)$ & $<.001$ \\
\hline Chemotherapy & $1.41(1.32-1.50)$ & $<.001$ & $1.02(0.97-1.06)$ & .44 \\
\hline Immunotherapy & $0.98(0.93-1.03)$ & .46 & $0.84(0.77-0.92)$ & $<.001$ \\
\hline
\end{tabular}

Abbreviations: HR, hazard ratio; LDH, lactate dehydrogenase; ULN, upper limit of normal.

OS advantage in stage IV disease, whereas it was inversely associated with OS in stage III disease. The discrepancy in rates of immunotherapy administration in stage IV cutaneous MMel between this study and another SEER population-based study can be ascribed to the inclusion of the study population between 2000 and 2009 in the SEER study. ${ }^{12}$ The approval of immune checkpoint inhibitors by the FDA in 2011 revolutionized the management of $\mathrm{MMel}$, showing promising results in improving survival. ${ }^{13}$ Since this approval, more T3 facilities may have started using checkpoint inhibitors in the management of MMel, leading to this robust $13 \%$ difference in use of immunotherapy. This possibility is supported by our findings, which showed that median OS of patients with stage IV disease treated at T3 facilities was much higher in those managed in 2012-2015 compared with those managed in 2004-2010.

Another possible explanation for better OS in T3 facilities is the availability of multidisciplinary care teams that can effectively handle treatment-related complications and have substantial experience in specialized multimodality approaches that are sometimes required for palliation and local disease control in metastatic cancers. In addition, it is more likely that patients treated at T3 facilities had better access to clinical trials of novel targeted agents and other newer modalities of therapy before their official FDA approval. Other 
nonmeasurable factors that may have impacted survival positively at T3 facilities include improved access to ancillary and supportive care services, as well as more resources for palliative care, which in turn has been associated with prolonged survival in advanced cancers. ${ }^{6,14}$

The study limitations are inherent to registry-based analyses. First, because of a lack of detailed information about the hospitals and specific type of therapies offered to patients (and their complications), we could not analyze specific reasons for improved survival in high-volume facilities compared with lower-volume facilities. Although one can argue that the difference in survival noted in our study may be attributed to the receipt of newer therapeutic modalities at T3 facilities, a survival advantage was evident even after adjusting for type of therapy received. Second, information on cancer-specific survival is lacking in the NCDB, and therefore patients treated at high-volume facilities may be healthier at baseline. To address this concern, we included the Charlson-Deyo comorbidity index score in the analysis, but did not see significant differences in this score among the facilities. Third, details are lacking on the percentage of patients enrolled in clinical trials among different facilities. It is likely that patients treated at high-volume facilities are more commonly enrolled in clinical trials. Fourth, the NCDB does not capture any data if patients switched institutions after receiving their first therapy. Because the NCDB data are obtained from Commission on Cancer (CoC)-accredited institutions only, the study results may not be applicable to some small rural facilities that are not CoC-accredited. Nevertheless, the NCDB provides real-world data that are not skewed by biases that arise from single institutional studies. Such a robust database is necessary for conducting volume-outcome studies effectively. Fifth, selection bias is possible in that facilities may tend to pick and choose their patients, which may potentially confound OS outcomes. Moreover, the financial cost differences and "network" issues encountered by patients in $\mathrm{T} 1$ versus $\mathrm{T} 3$ facilities may have played a role in access to care, and thus the OS differences noticed in this analysis. To address this concern, we included health insurance and socioeconomic status in the multivariate analysis and showed that patients with private insurance and higher income had better median OS compared with their counterparts. Finally, the median number of cases cutoff we used for classifying a facility as high-volume requires further validation, and no definite conclusions may be drawn regarding whether a specific type of highvolume facility should be the norm for optimal cancer care delivery.

Despite these limitations, the study results present several important considerations. Note that not all patients with advanced MMel have access to high-volume facilities, for multiple reasons that may include financial burden and the ability to travel long distances. This study sheds light on the practical "care disparities" among patients with advanced cutaneous MMel and emphasizes the need for recognizing the differences in cancer care delivery models that may help improve survival outcomes at lower-volume treatment facilities.

\section{Conclusions}

After adjusting for patient demographics, socioeconomic status (median household income, education level, insurance), treatment modality, and Charlson-Deyo comorbidity index score, patients treated at T3 facilities that handle $\geq 19$ and $\geq 10$ cases per year of stage III and IV cutaneous MMel, respectively, had a significantly lower risk of allcause mortality. Patients with stage IV MMel treated at T3 facilities were more likely to receive immunotherapy and chemotherapy. These results underscore the need for adaptation of best practices at lower-volume facilities to improve patient outcomes.

Submitted February 15, 2019; accepted for publication May 16, 2019

Previous presentation: This work was presented at the 2018 ASCO Annual Meeting; June 1-5, 2018; Chicago, Illinois.

Author contributions: Study concept and design: Tell, Kommalapati. Data acquisition, analysis, or interpretation: All authors. Manuscript-draft: Kommalapati, Tella. Manuscript-critical revision: All authors. Supervision: Ganti, Marr. Dr. Kommalapati had full access to the study data and takes responsibility for the integrity of the data and the accuracy of the data analysis.

Disclosures: The authors have not received any financial consideration from any person or organization to support the preparation, analysis, results, or discussion of this article.

Correspondence: Anuhya Kommalapati, MBBS, Department of Oncology, H. Lee Moffitt Cancer Center, Tampa, FL 33612. Email: anuhya781@gmail.com

\section{References}

1. National Cancer Institute. Cancer Stat Facts: Melanoma of the Skin. Available at: https://seer.cancer.gov/statfacts/html/melan.html. Accessed March 2, 2018

2. Maverakis E, Cornelius LA, Bowen GM, et al. Metastatic melanoma-a review of current and future treatment options. Acta Derm Venereol 2015; 95:516-524.

3. Halm EA, Lee C, Chassin MR. Is volume related to outcome in health care? A systematic review and methodologic critique of the literature. Ann Intern Med 2002;137:511-520.

4. Begg CB, Cramer LD, Hoskins WJ, et al. Impact of hospital volume on operative mortality for major cancer surgery. JAMA 1998;280: 1747-1751.

5. Haj Mohammad N, Bernards N, Besselink MG, et al. Volume matters in the systemic treatment of metastatic pancreatic cancer: a population-based study in the Netherlands. J Cancer Res Clin Oncol 2016;142:1353-1360.

6. Haj Mohammad N, Bernards N, van Putten M, et al. Volume-outcome relation in palliative systemic treatment of metastatic oesophagogastric cancer. Eur J Cancer 2017;78:28-36. 
7. Mokdad AA, Zhu H, Marrero JA, et al. Hospital volume and survival after hepatocellular carcinoma diagnosis. Am J Gastroenterol 2016;111: 967-975.

8. Kumachev A, Trudeau ME, Chan KK. Associations among socioeconomic status, patterns of care and outcomes in breast cancer patients in a universal health care system: Ontario's experience. Cancer 2016;122:893-898.

9. Quaglia A, Lillini R, Mamo C, et al. Socio-economic inequalities: a review of methodological issues and the relationships with cancer survival. Crit Rev Oncol Hematol 2013;85:266-277.

10. Aarts MJ, Lemmens VE, Louwman MW, et al. Socioeconomic status and changing inequalities in colorectal cancer? A review of the associations with risk, treatment and outcome. Eur J Cancer 2010;46:2681-2695.
11. Suneja G. The impact of socioeconomic status on cancer care. Available at: https://www.oncolink.org/conferences/coverage/asco/oncolink-atasco-2011/monday-june-6-2011/the-impact-of-socioeconomic-statuson-cancer-care-and-survival. Accessed May 22, 2019.

12. Huo J, Lairson DR, Du XL, et al. Hospital case volume is associated with improved survival for patients with metastatic melanoma. Am J Clin Oncol 2016:39:491-496.

13. Achkar T, Tarhini AA. The use of immunotherapy in the treatment of melanoma. J Hematol Oncol 2017;10:88

14. Meier DE. Increased access to palliative care and hospice services: opportunities to improve value in health care. Milbank Q 2011;89: 343-380. 